\title{
Direct Quantification of Quasi-Fermi-Level Splitting in Organic Semiconductor Devices
}

\author{
Drew B. Riley®,$^{1,}{ }^{*}$ Oskar J. Sandberg $\odot,{ }^{1, \dagger}$ Nora M. Wilson $\odot,{ }^{2}$ Wei Li, ${ }^{1}$ Stefan Zeiske, ${ }^{1}$ \\ Nasim Zarrabi, ${ }^{1}$ Paul Meredith, ${ }^{1}$ Ronald Österbacka $\odot,{ }^{2}$ and Ardalan Armin ${ }^{1, \sharp}$ \\ ${ }^{1}$ Sustainable Advanced Materials Programme (Sêr SAM), Department of Physics, Swansea University, Singleton \\ Park, Swansea SA2 8PP, United Kingdom \\ ${ }^{2}$ Faculty of Science and Engineering, Abo Akademi University, 20500 Turku, Finland
}

(Received 5 March 2021; accepted 7 May 2021; published 15 June 2021)

\begin{abstract}
Nonradiative losses to the open-circuit voltage are a primary factor in limiting the power-conversion efficiency of organic photovoltaic devices. The dominate nonradiative loss in the bulk is intrinsic to the active layer and can be determined from the quasi-Fermi-level splitting (QFLS) and the radiative thermodynamic limit of the photovoltage. Quantification of the QFLS in thin-film devices with low mobility is challenging due to the excitonic nature of photoexcitation and additional sources of nonradiative loss associated with the device structure. This work outlines an experimental approach based on electromodulated photoluminescence, which can be used to directly measure the intrinsic nonradiative loss to the opencircuit voltage, thereby quantifying the QFLS. Drift-diffusion simulations are carried out to show that this method accurately predicts the QFLS in the bulk of the device regardless of device-related nonradiative losses. State-of-the-art PM6:Y6-based organic solar cells are used as a model to test the experimental approach and the QFLS is quantified and shown to be independent of device architecture. This work provides a method to quantify the QFLS of organic solar cells under operational conditions, fully characterizing the different contributions to the nonradiative losses of the open-circuit voltage. The reported method will be useful not only in characterizing and understanding losses in organic solar cells but also in other device platforms such as light-emitting diodes and photodetectors.
\end{abstract}

DOI: 10.1103/PhysRevApplied.15.064035

\section{INTRODUCTION}

As power-conversion efficiencies of organic solar cells surpass $18 \%[1,2]$, it has become essential to comprehend and eradicate every mechanism contributing to efficiency reduction. Losses to the short-circuit current $\left(J_{\mathrm{SC}}\right)$ have been minimized by increasing charge generation and collection through the use of nonfullerene acceptors, resulting in short-circuit currents consistently above $20 \mathrm{~mA} / \mathrm{cm}^{2}$ [2-5]. While losses to the open-circuit voltage $\left(V_{\mathrm{OC}}\right)$ have also decreased with nonfullerene acceptors the mechanisms contributing to $V_{\mathrm{OC}}$ losses are not as straightforward to interpret as those contributing to the short-circuit current

\footnotetext{
*1915821@swansea.ac.uk

†o.j.sandberg@swansea.ac.uk

+ardalan.armin@swansea.ac.uk
}

Published by the American Physical Society under the terms of the Creative Commons Attribution 4.0 International license. Further distribution of this work must maintain attribution to the author(s) and the published article's title, journal citation, and DOI. losses. As losses in the $V_{\mathrm{OC}}$ due to radiative recombination channels are unavoidable in solar cells, the radiative limit to the $V_{\mathrm{OC}}\left(V_{\mathrm{OC}}^{\mathrm{rad}}\right)$ is considered the primary benchmark to target. Nonradiative losses to the $V_{\mathrm{OC}}$ result in proportional decreases to the power-conversion efficiency, making their detection and suppression a high research priority [613]. Nonradiative recombination losses can be intrinsic to the active layer of the device due to the interaction of electronic states with the surrounding medium via vibrational states. Additionally, nonradiative losses can occur at the interfaces between the active layer and the electrode (or interlayer). This nonradiative recombination channel, defined by the extraction of minority carriers out of the active layer at the "wrong" contact (i.e., electrons at the anode, holes at the cathode), is often referred to as surface recombination [14-22]. As the $V_{\mathrm{OC}}$ is ultimately defined by the quasi-Fermi-level splitting (QFLS) of electrons and holes in the device (at open circuit), an accurate quantification of the QFLS is key for understanding nonradiative recombination processes in photovoltaic devices.

Quasi-Fermi levels and the QFLS, as introduced by Shockley, are essential concepts used to describe nonequilibrium steady-state operation of electronic 
semiconductors [23]. This concept has been expanded by Würfel for optoelectronic processes by including a chemical potential associated with the radiative process [24]. Würfel has shown that the chemical potential of an absorbed or emitted photon $\left(\mu_{\gamma}\right)$ is equal to the QFLS in the semiconductor. Equipped with this understanding, researchers have successfully employed photoluminescence measurements to evaluate the QFLS in systems where absorption and emission are dominated by free carriers [24-26] and have subsequently applied this technique to organic semiconductors [27-29]. Regrettably, photoexcitation in organic semiconductors is not dominated by free carriers but by Coulombically bound singlet excitonic states [30]. To generate free carriers, excitons must diffuse to a donor-acceptor interface and form intermediary charge-transfer (CT) states, which primarily decay nonradiatively [31]. While CT states may be in equilibrium with free carriers, excitons are generally not and may decay radiatively before forming a CT state [4,6-9]. Therefore, traditional photoluminescence measurements (as applied to inorganic semiconductors) are not valid as a method of determining the QFLS in organic semiconductor blends, since the contribution to charge generation is simultaneously overestimated for excitons and underestimated for CT states. These discrepancies can be circumvented by employing Rau's reciprocity principle between the charge collection of photogenerated carriers (under illumination) and the electroluminescent emission in the dark. Rau's theory assesses the nonradiative losses by providing an expression for both the radiative limit and the nonradiative losses of the open-circuit voltage of a device [32]. This has been successfully employed in conjunction with electroluminescent external quantum efficiency measurements to quantify the QFLS and related losses in a wide variety of semiconductor-based solar cells [33-36]. However, the corresponding $V_{\mathrm{OC}}$ loss derived from this approach generally includes contributions from both intrinsic bulkrelated processes and surface recombination. In organic solar cells, the presence of surface recombination is usually correlated with electrode-induced photovoltage losses at the contacts [37-39], causing a mismatch between $V_{\mathrm{OC}}$ and the associated QFLS in the bulk. As such, measurements based on electroluminscence cannot differentiate between intrinsic and electrode-induced photovoltage losses, leading to a consistent underestimation of the QFLS in organic photovoltaic devices. A method for overcoming the difficulty in quantifying the QFLS has recently been suggested using photoinduced absorption [39]. However, this method requires detailed knowledge about the absorption cross section and charge-transport parameters of the device, which must necessarily be semitransparent. A more direct quantification of the QFLS in an optimized device would serve well to complement this method and provide quantification of the QFLS in devices where photoinduced absorption is not trivial.
The work described herein establishes an experimental approach termed electromodulated-photoluminescence quantum efficiency, providing a pathway to measuring the intrinsic nonradiative losses occurring within the active layer of an organic solar cell at open-circuit conditions. Through the principle of reciprocity, this technique quantifies the QFLS in the active layer and subsequently the electrode-induced voltage losses of a solar cell under operational conditions. Drift-diffusion simulations are employed to simulate the proposed experiment and compare to the computable QFLS [40-46]. Further, PM6:Y6 (for a list of the chemical acronyms, see Sec. SIII of the Supplemental Material [47]) solar cells have been fabricated with various contacts designed to increase the nonradiative loss without modifying the QFLS in the bulk to confirm the simulated experiments. The electromodulated-photoluminescence measurements have been found to successfully predict the QFLS and related losses over the range of devices used.

\section{THEORETICAL BACKGROUND}

For a semiconductor with flat featureless quasi-Fermi levels, the net emission flux of photons from the surface $\left(\Phi_{\mathrm{em}}\right)$ at energy $E_{\gamma}$ is determined by the chemical potential of radiation $\mu_{\gamma}$ via [24]

$$
\Phi_{\mathrm{em}}\left(E_{\gamma}\right)=a\left(E_{\gamma}\right) \Phi_{\mathrm{BB}}\left(E_{\gamma}\right)\left[\exp \left(\frac{\mu_{\gamma}}{k_{B} T}\right)-1\right],
$$

assuming that $E_{G}-\mu_{\gamma} \gg k_{B} T$, where $E_{G}$ is the band gap of the semiconductor, $k_{B}$ is the Boltzmann constant, $T$ is the temperature of the lattice, $a\left(E_{\gamma}\right)$ is the spectral absorbance, and $\Phi_{\mathrm{BB}}\left(E_{\gamma}\right)$ is the spectral black-body radiation at room temperature. Using the reciprocity principle, an analogous relation between the emitted radiation, the photovoltaic external quantum efficiency $\left(\mathrm{EQE}_{\mathrm{PV}}\right)$, and the voltage $(V)$ can be expressed as [32]

$$
\Phi_{\mathrm{em}}\left(V, E_{\gamma}\right)=\mathrm{EQE}_{\mathrm{PV}}\left(E_{\gamma}\right) \Phi_{\mathrm{BB}}\left(E_{\gamma}\right)\left[\exp \left(\frac{q V}{k_{B} T}\right)-1\right],
$$

where $q$ is the elementary charge. Note that the substitution of $\mathrm{EQE}_{\mathrm{PV}}$ for $a$ and $q V$ for $\mu_{\gamma}$ in Eq. (2) produces Eq. (1). However, this substitution is only valid when the charge carriers in the semiconductor are in equilibrium with the emitted radiation across the device structure, which is satisfied when the quasi-Fermi levels are flat across the junction $\left(V=V_{\mathrm{OC}}\right)$ and between the electrical contacts [24]. Further, Eqs. (1) and/or (2) may become invalid in the presence of nonequilibrium states such as radiativetrap states [48] or nonequilibrium excitons (as discussed above). 
The radiative limit to the $V_{\mathrm{OC}}$ can be calculated from detailed balance as [32]

$$
V_{\mathrm{OC}}^{\mathrm{rad}}=\frac{k_{B} T}{q} \ln \left[\frac{J_{\mathrm{ph}}}{J_{0}^{\mathrm{rad}}}+1\right],
$$

where $J_{\mathrm{ph}}$ is the photocurrent generated at one-sun illumination $\left(J_{\mathrm{ph}}=q \int_{0}^{\infty} \mathrm{EQE}_{\mathrm{PV}} \Phi_{\text {sun }} d E_{\gamma}\right)$ and $J_{0}^{\mathrm{rad}}$ is the radiative dark saturation current $\left(J_{0}^{\mathrm{rad}}=q \int_{0}^{\infty} \mathrm{EQE}_{\mathrm{PV}} \Phi_{\mathrm{BB}} d E_{\gamma}\right)$. The true open-circuit voltage is the difference between the radiative limit and the total nonradiative losses $\left(\Delta V_{\mathrm{OC}}^{\mathrm{NR}, \text { total }}\right)$, defined as the sum of the intrinsic $\left(\Delta V_{\mathrm{OC}}^{\mathrm{NR} \text {,intrinsic }}\right)$ and electrode-induced $\left(\Delta V_{\mathrm{OC}}^{\mathrm{NR} \text {,electrode }}\right)$ losses:

$$
q V_{\mathrm{OC}}=q V_{\mathrm{OC}}^{\mathrm{rad}}-q \Delta V_{\mathrm{OC}}^{\mathrm{NR}, \text { total }} .
$$

The QFLS ( $\left.q V_{\mathrm{QFLS}}\right)$ in the bulk of the active layer, which is ideally equal to $\mu_{\gamma}$, is then determined by the difference between the radiative limit and the intrinsic nonradiative losses:

$$
q V_{\mathrm{QFLS}}=q V_{\mathrm{OC}}^{\mathrm{rad}}-q \Delta V_{\mathrm{OC}}^{\mathrm{NR}, \text { intrinsic }} .
$$

In general, the nonradiative losses of the $V_{\mathrm{OC}}$ can be calculated from the external quantum efficiency $\eta_{\text {LED }}$ of a device operated as a light-emitting diode (LED), defined as the ratio of the emitted radiation to the injected current $\left(\eta_{\mathrm{LED}}=q \Phi_{\mathrm{em}} / J_{\mathrm{inj}}\right)$ :

$$
q \Delta V_{\mathrm{OC}}^{\mathrm{NR}}=-k_{B} T \ln \left[\eta_{\mathrm{LED}}\right] .
$$

In order to isolate the effects of intrinsic and electrodeinduced nonradiative loss, one must consider the experimental conditions under which $\eta_{\text {LED }}$ is measured as well as the relation between $\Phi_{\mathrm{em}}$ and Eqs. (1) and (2). Direct probing of the emission described in Eq. (1) would, in the ideal case, allow for the quantification of the intrinsic nonradiative losses to the $V_{\mathrm{OC}}$ and subsequently the QFLS.

\section{EXPERIMENT}

Figure 1 shows two experimental apparatuses used to measure $\eta_{\mathrm{LED}}$, the traditional electroluminescence [Fig. 1(a)] and the alternative electromodulated photoluminescence [Fig. 1(c)]. During electroluminescence measurements, the device under test is held in the dark and a square-wave modulated voltage is applied to the device in forward bias by the function generator (Keysight 33500B). The current response can be measured as a square wave on the oscilloscope (Rohde \& Schwarz RTM3004) and the resulting electroluminescence is captured by a $50-\mathrm{mm}$ lens, filtered with three low-pass filters (Thorlabs FEL 650, 600,550 ) (necessary to remove scattered pump light in the subsequent experiment), amplified by a silicon photoreceiver (FEMTO OE-300-Si-30), and measured with a lock-in amplifier (Stanford Research Systems SR860). The resulting external quantum efficiency $\left(\eta_{\mathrm{EL}}\right)$ can be calculated from the ratio of the electroluminescence intensity to the injected current at the reference frequency. This apparatus suffers from reduced geometric and spectral light-collection efficiency, which can be compensated for by calibrating the apparatus to an absolute measurement of $\eta_{\mathrm{EL}}$ (see Sec. SIII). To estimate $\eta_{\mathrm{EL}}$ at a condition similar to open-circuit one-sun illumination, it is conventional to choose an injected dark current equal to the short-circuit current at one sun $\left(J_{\mathrm{inj}}^{\mathrm{dark}}=J_{\mathrm{SC}}^{\mathrm{AM} 1.5}\right)$. In contrast to Fig. 1(a), Fig. 1(c) shows the electromodulated-photoluminescence apparatus. Prior to performing an electromodulatedphotoluminescence measurement, the sample is illuminated with a laser (custom-made 520-nm diode laser) and the short-circuit current $\left(J_{\mathrm{SC}}\right)$ is measured. To perform the electromodulated-photoluminescence quantum yield measurements, the device is brought to open-circuit conditions, where the applied time-dependent voltage, supplied by the function generator at angular frequency $\omega_{V}$, the resulting injected current, measured by the oscilloscope, and the emitted photoluminescence have the form

$$
\begin{aligned}
V_{\text {app }}(t) & =V_{\text {off }}+\Delta V \sin \left(\omega_{V} t\right) \\
J_{\text {inj }}(t) & =\Delta J \sin \left(\omega_{V} t\right) \\
\Phi_{\text {em }}(t) & =\Phi_{0}+\Delta \Phi \sin \left(\omega_{V} t\right),
\end{aligned}
$$

where $\Delta J$ is kept smaller than $0.1 J_{\mathrm{SC}}$ by adjusting $\Delta V$ in order to keep the change in emission linear with the change in applied voltage (for details related to the size of this perturbation, see Sec. SIII of the Supplemental Material [47]) and $V_{\text {off }}$ is set such that the mean current is zero. The resulting luminescence current amplitude $(\Delta \Phi)$ is collected, amplified, and measured in the same manner as the electroluminescence measurement described above. The electromodulated-photoluminescence quantum efficiency is defined as the ratio between the luminescence intensity measured on the photodetector and the injected current amplitudes:

$$
\eta_{\mathrm{EMPL}}=\frac{q \Delta \Phi}{\Delta J}
$$

To evaluate $\eta_{\text {EMPL }}$ at conditions close to open-circuit onesun illumination, the laser power is adjusted such that the short-circuit current equals $J_{\mathrm{SC}}^{\mathrm{AM} 1.5}$, leading $V_{\text {off }}$ to be approximately $V_{\mathrm{OC}}^{\mathrm{AM} 1.5}$. As this experiment has the same spectral and geometric light-collection efficiency as the electroluminescence measurement, the absolute $\eta_{\mathrm{EMPL}}$ is found by multiplying by the same calibration factor.

\section{RESULTS AND DISCUSSION}

Time-domain and steady-state drift-diffusion simulations (details are given in Sec. SI of the Supplemental 


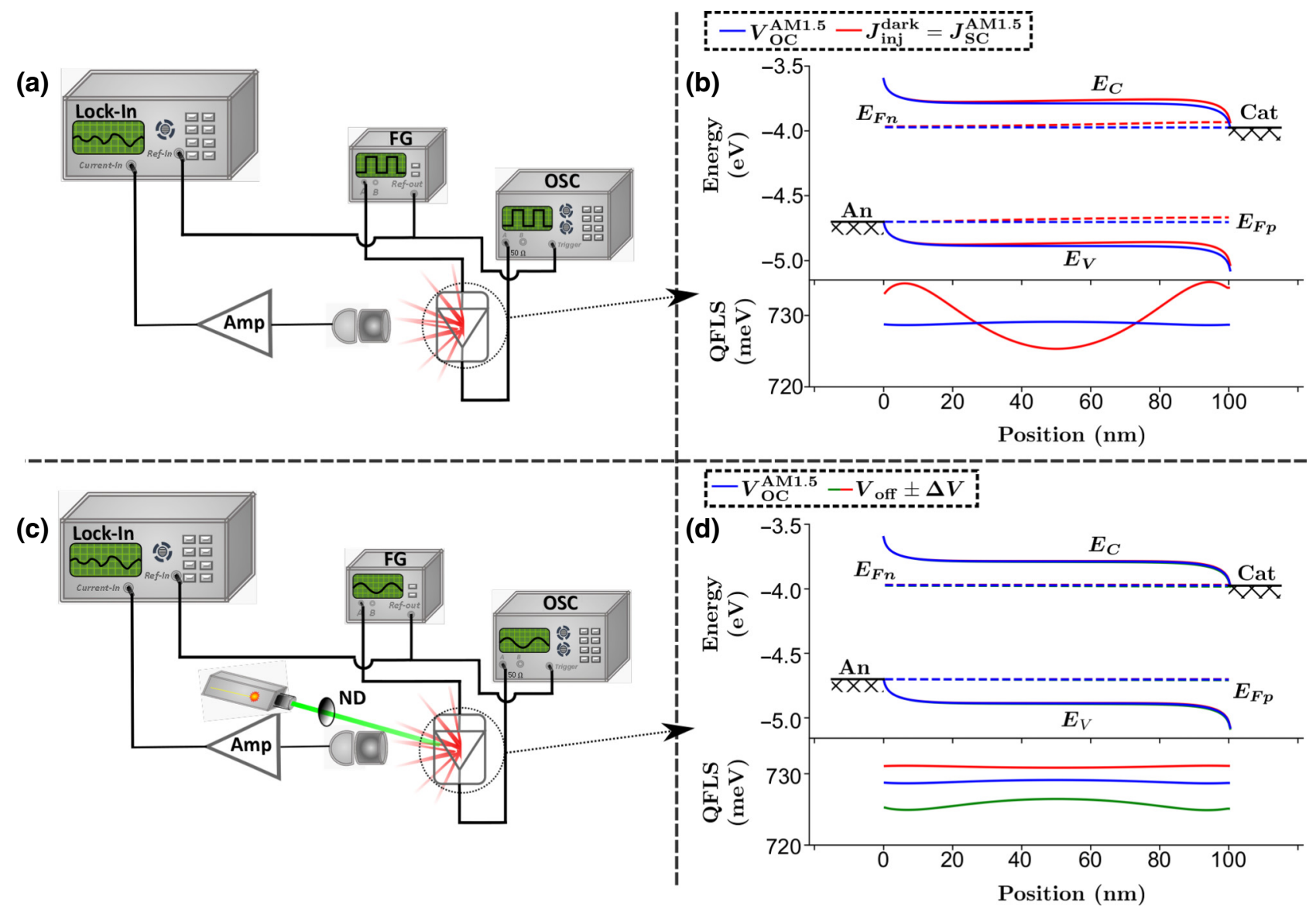

FIG. 1. (a) The electroluminescence schematic. (b) The simulated conduction $\left(E_{C}\right)$ and valence levels $\left(E_{V}\right)$ (solid lines), electron $\left(E_{F n}\right)$ and hole $\left(E_{F p}\right)$ quasi-Fermi levels (dashed lines), and QFLS under the following conditions: one-sun open-circuit (blue) and dark injected current of one-sun short-circuit current (red). (c) The electromodulated-photoluminescence schematic. (d) The simulated conduction, valence, electron and hole quasi-Fermi levels, and QFLS under the following conditions: one-sun open-circuit (blue) and one-sun $V_{\text {off }} \pm \Delta V$ (red and green). An, anode; Cat, cathode; FG, function generator; Amp, current amplifier; OSC, oscilloscope; ND, neutral-density wheel.

Material [47]) are employed to demonstrate the difference between electroluminescence and electromodulatedphotoluminescence measurements in idealized systems before undertaking experiments. Figures 1(b) and 1(d) compare the simulated conduction, valence, electron and hole quasi-Fermi levels, and QFLS across a device under one-sun open-circuit (blue), electroluminescence [Fig. 1(b), red], and electromodulated-photoluminescence [Fig. 1(d), red and green] conditions (for details of the electromodulated-photoluminescence simulations, see Sec. SII of the Supplemental Material [47]). Under electroluminescence conditions, the conduction and valence levels, along with the quasi-Fermi levels, deviate from the one-sun open-circuit conditions as the QFLS varies across the device. In contrast, under the maximum and minimum voltages applied during electromodulated photoluminescence, the conduction and valence levels, and the quasi-Fermi levels, are indistinguishable from those of one-sun open-circuit conditions. This indicates that electromodulated-photoluminescence conditions are closer to operational conditions than electroluminescence conditions. Crucially, under electromodulated-photoluminescence conditions, the QFLS varies little across the device for each voltage and much less over the applied voltage range compared with the $10-\mathrm{meV}$ variance in QFLS under $J_{\text {inj }}^{\text {dark }}=J_{\mathrm{SC}}^{\mathrm{AM} 1.5}$ conditions, seen in panel (b). The small variation in QFLS indicates that under electromodulated-photoluminescence conditions, the device is very nearly in equilibrium with the emitted radiation, suggesting that the emission is described by Eq. (1) and therefore can be used to quantify the QFLS, while the emission under electroluminescence conditions is given by Eq. (2).

To explore the relationship between QFLS and $V_{\mathrm{OC}}$, steady-state simulations of devices under open-circuit conditions are conducted. By increasing the electron injection barrier $\left(\phi_{n, \text { cat }}\right)$ at the cathode, the effect of an increased electrode-induced photovoltage loss can be simulated for 


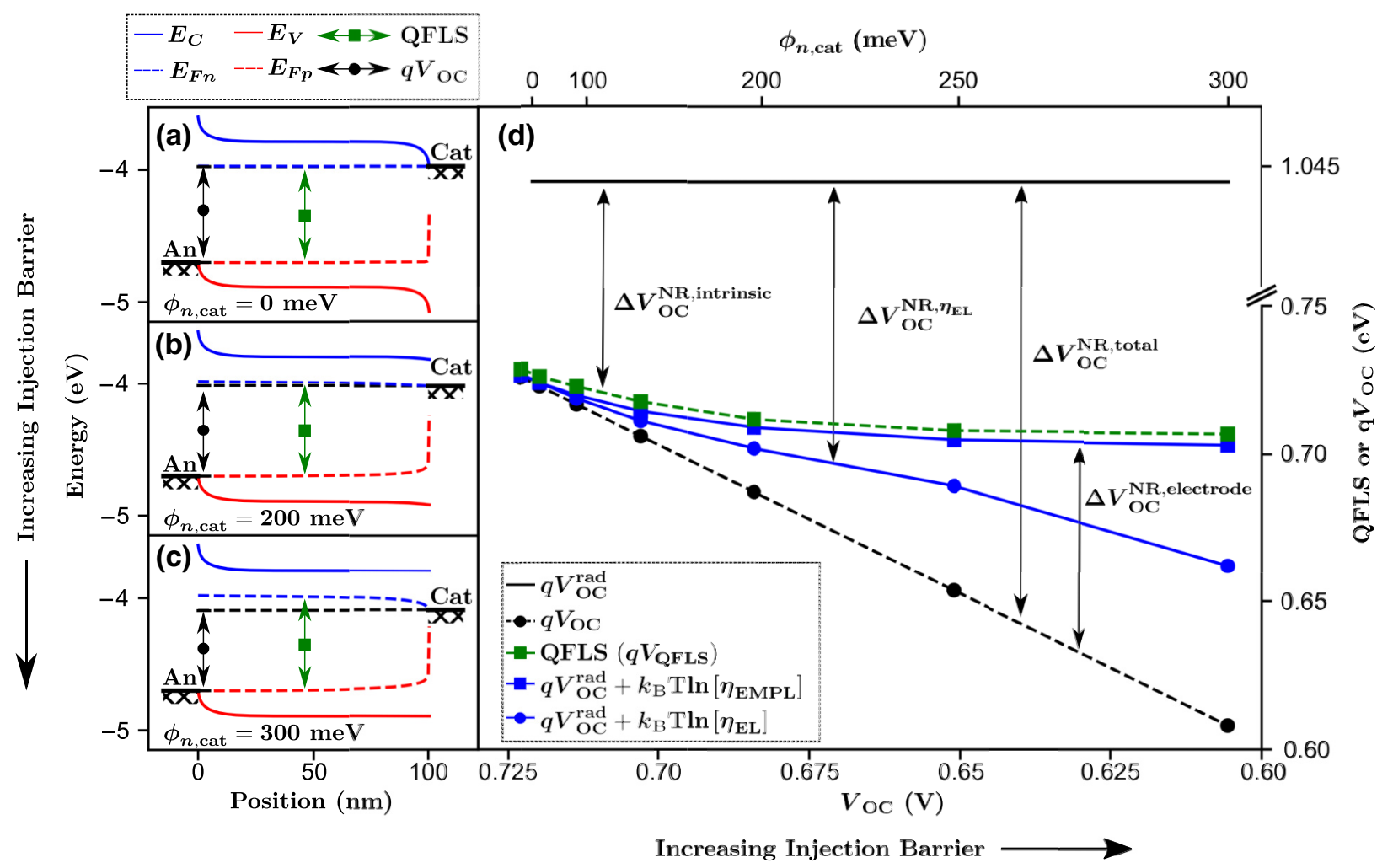

FIG. 2. The simulated (a)-(c) conduction $\left(E_{C}\right)$, valence $\left(E_{V}\right)$, electron $\left(E_{F n}\right)$ and hole $\left(E_{F p}\right)$ quasi-Fermi levels for devices with (a) no injection barrier, (b) a medium injection barrier, and (c) a high injection barrier. (d) The simulated injection barrier dependence of $V_{\mathrm{OC}}$ (black circles), QFLS (green squares), and nonradiative losses measured by simulating electroluminescence (blue circles) and electromodulated-photoluminescence (blue squares) experiments.

organic photovoltaic devices [37,38]. Figures 2(a)-2(c) show the energy levels for devices with (a) no, (b) small, and (c) large injection barriers. The QFLS is defined as the difference between the electron and hole quasi-Fermi levels in the bulk of the device (here, taken to mean the exact center) as indicated by the green arrow, while the $V_{\mathrm{OC}}$ can be determined by the difference between electrode Fermi-levels as indicated by the black arrows [37]. As $\phi_{n, \text { cat }}$ is increased, the electron quasi-Fermi level near the cathode has to curve down in order to maintain equilibrium with the cathode work function. This leads to a considerable gradient in the electron quasiFermi level near the cathode, ultimately reducing the $V_{\mathrm{OC}}$, while the QFLS inside the bulk remains predominately unaffected. Figure 2(d) summarizes these data for devices with electron injection barriers between 0 and $300 \mathrm{meV}$. In the absence of an injection barrier, the QFLS and $q V_{\mathrm{OC}}$ are identical; however, as the injection barrier is increased, the $V_{\mathrm{OC}}$ reduces while the QFLS is weakly affected. This is consistent with previous work, where it has been shown that $q V_{\mathrm{OC}} \propto E_{G}-\phi_{n \text {,cat }}$ for a large enough electron injection barrier at the cathode [37,49]. The size of the intrinsic and total nonradiative losses as well as those induced by the electrode $\left(\Delta V_{\mathrm{OC}}^{\mathrm{NR}, \text { electrode }}\right)$ and predicted by electroluminescence $\left(\Delta V_{\mathrm{OC}}^{\mathrm{NR}, \eta_{\mathrm{EL}}}\right)$ are labeled for clarity.

To uncover the relationship between the QFLS and $\eta_{\mathrm{LED}}$, electroluminescence and electromodulatedphotoluminescence experiments are simulated for each system. The nonradiative losses, calculated from Eq. (6), for both electroluminescence and electromodulated photoluminescence are subtracted from the radiative limit; the blue curves in Fig. 2(d) show the results of these calculations. The predicted losses from electroluminescence, when subtracted from $V_{\mathrm{OC}}^{\mathrm{rad}}$, follow $q V_{\mathrm{OC}}$ for devices with a low injection barrier, as expected from the reciprocity principle expressed in terms of Eq. (2). A discrepancy between the expected $q V_{\mathrm{OC}}$ given by electroluminescence and the actual $q V_{\mathrm{OC}}$ is seen for devices with a large injection barrier. We note that an increased injection barrier at the cathode (reducing the number of injected electrons) generally makes the overall charge in the device uneven, which might explain the divergence from the expectations of the reciprocity principle. In contrast, the simulated losses obtained by electromodulated photoluminescence follow the QFLS across all devices. This indicates that electromodulated photoluminescence is probing the intrinsic losses occurring within the active layer of the device, 
which is useful for discerning material properties such as QFLS and optimizing electrodes in low-mobility systems, such as organic photovoltaic devices. On the other hand, electroluminescence is sensitive to both the intrinsic and electrode-induced nonradiative losses, which is useful in determining total nonradiative losses in an optimized device. Consequentially, based on this analysis, the open-circuit voltage expected from reciprocity $V_{\mathrm{oc}, \mathrm{EL}}$ and the QFLS ( $\left.q V_{\mathrm{QFLS}}\right)$ in the bulk can be calculated from Eqs. (4)-(6) as

$$
\begin{aligned}
& q V_{\mathrm{oc}, \mathrm{EL}}=q V_{\mathrm{OC}}^{\mathrm{rad}}+k_{B} T \ln \left[\eta_{\mathrm{EL}}\right], \\
& q V_{\mathrm{QFLS}}=q V_{\mathrm{OC}}^{\mathrm{rad}}+k_{B} T \ln \left[\eta_{\mathrm{EMPL}}\right] .
\end{aligned}
$$

To validate this proposal and the simulations (shown in Fig. 2), devices with cathode materials of different work functions are prepared to emulate the effect of varying the electron injection barrier at the cathode. Each device is made with ITO/PEDOT:PSS as the anode and a 100nm-PM6:Y6 active layer, while the cathode is varied to alter the electrode-induced nonradiative losses. Using this architecture, an optimized device with a power-conversion efficiency of $15.3 \%$ is created, using $\mathrm{PDINO} / \mathrm{Ag}$ as the cathode. Figure 3 shows the current-voltage characteristics for each device. Despite a relatively small shift in $J_{\mathrm{SC}}$ and the fill factor between the different devices, the powerconversion efficiency is reduced due to the reduction in $V_{\mathrm{OC}}$, indicative of increasing electrode-induced nonradiative losses due to surface recombination [see Fig. S3(a) of the Supplemental Material [47] and Refs. [37,38]]. While the optimized device has a $V_{\mathrm{OC}}$ of $0.847 \mathrm{~V}$, the device using a silver-only cathode exhibits a $V_{\mathrm{OC}}$ of $0.787 \mathrm{~V}$; the added interlayers (PDNIO or PFN-Br) modify the work function to create a more Ohmic cathode. Figures 4(a)-4(g) show the device structures along with a sketch of the quasi-Fermi levels at open-circuit conditions. A near-Ohmic cathode [panels (a) and (b)] will cause the QFLS in the bulk and the

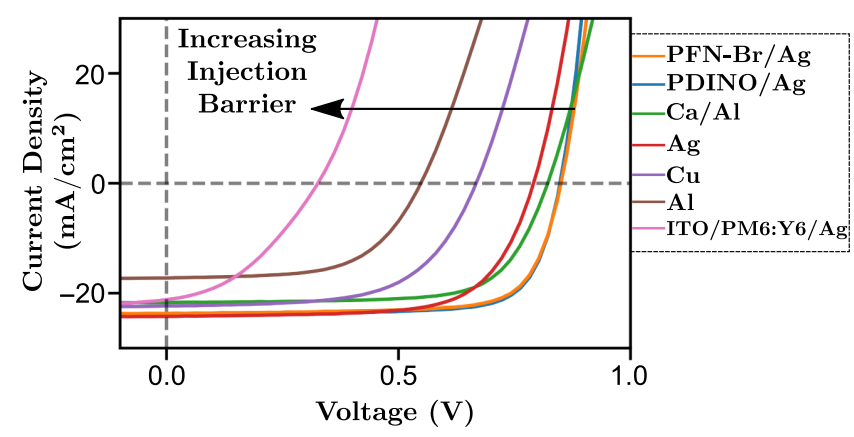

FIG. 3. The current-density-voltage curves at one-sun illumination for devices with various cathode materials. All devices have a structure ITO/PEDOT:PSS/PM6:Y6/cathode (cathode material shown in the legend), except for the pink curve, where the device structure is ITO/PM6:Y6/Ag. $q V_{\mathrm{OC}}$ to be roughly equivalent, due to the small injection barrier. Moving down from panel (a) to panel (f), the injection barrier is increased by increasing the cathode work function, causing a decrease in the $V_{\mathrm{OC}}$ while the QFLS in the bulk of the active layer remains unchanged. The loss to the $V_{\mathrm{OC}}$ is due to the increasing electrode-induced nonradiative loss associated with the increasing injection barrier. Figures 4(h)-4(m) show the LED external quantum efficiency measured by both electroluminescence and electromodulated photoluminescence for each device as a function of the dark injected current density or the shortcircuit current density, respectively. As the injection barrier is increased, $\eta_{\mathrm{EL}}$ decreases until it is undetectable, while $\eta_{\text {EMPL }}$ does not change substantially. This suggests that $\eta_{\mathrm{EMPL}}$ is measuring losses intrinsic to the semiconductor, while $\eta_{\mathrm{EL}}$ is influenced by the structure of the device, as predicted by the simulations in Fig. 2. Figures 4(g) and 4(n) show the structure and external quantum efficiencies for a device without anode or cathode interlayers, severely limiting the driving force for charge extraction by creating an additional injection barrier at the anode. While electroluminescence emission is not measurable in this case, the corresponding electromodulated-photoluminescence emission is. In this low- $V_{\mathrm{OC}}$ device, $\eta_{\mathrm{EMPL}}$ is similar to that of the other devices. This confirms that electromodulatedphotoluminescence measurements are insensitive to device structure and are measuring an intrinsic property of the active layer.

The radiative limit to $V_{\mathrm{OC}}$ is calculated by measuring the photovoltaic external quantum efficiency on the optimized device and is found to be $1.077 \mathrm{~V}$ (see Sec. SIII of the Supplemental Material [47]). The expected $V_{\mathrm{OC}}$ is calculated from $\eta_{\mathrm{EL}}$ at $J_{\mathrm{Inj}}^{\mathrm{dark}}=J_{\mathrm{SC}}^{\mathrm{AM} 1.5}$ in accordance with Eq. (11) and the QFLS is calculated from $\eta_{\mathrm{EMPL}}$ at $J_{\mathrm{SC}}=J_{\mathrm{SC}}^{\mathrm{AM} 1.5}$, in accordance with Eq. (12). Figure 5(a) shows the QFLS, the expected $q V_{\mathrm{OC}}$, and the measured $q V_{\mathrm{OC}}$ at one-sun conditions for devices with increasing nonradiative losses. The black arrows indicate the magnitudes of the various nonradiative losses as guides for the eye. Figures 5(b)-5(d) show examples of device structures with small (b), medium (c), and large (d) injection barriers as a guide. The expected $q V_{\mathrm{OC}}$ based on the measured electroluminescence follows the actual $q V_{\mathrm{OC}}$ for devices with negligible injection barriers and occupies the region between the QFLS and $q V_{\mathrm{OC}}$ for devices with impractical injection barriers, as predicted by the simulations in Fig. 2. The calculated QFLS is constant throughout all devices as expected, demonstrating the precision of the method, and consistent with a previous study on the same material system using photoinduced absorption, further exemplifying the accuracy [39].

The inset of Fig. 5(a) enhances the devices with the lowest nonradiative losses. In the $\mathrm{PDINO} / \mathrm{Ag}$ device $\left(V_{\mathrm{OC}}=0.847 \mathrm{~V}\right)$, the QFLS is $853 \pm 5 \mathrm{meV}$, while the 


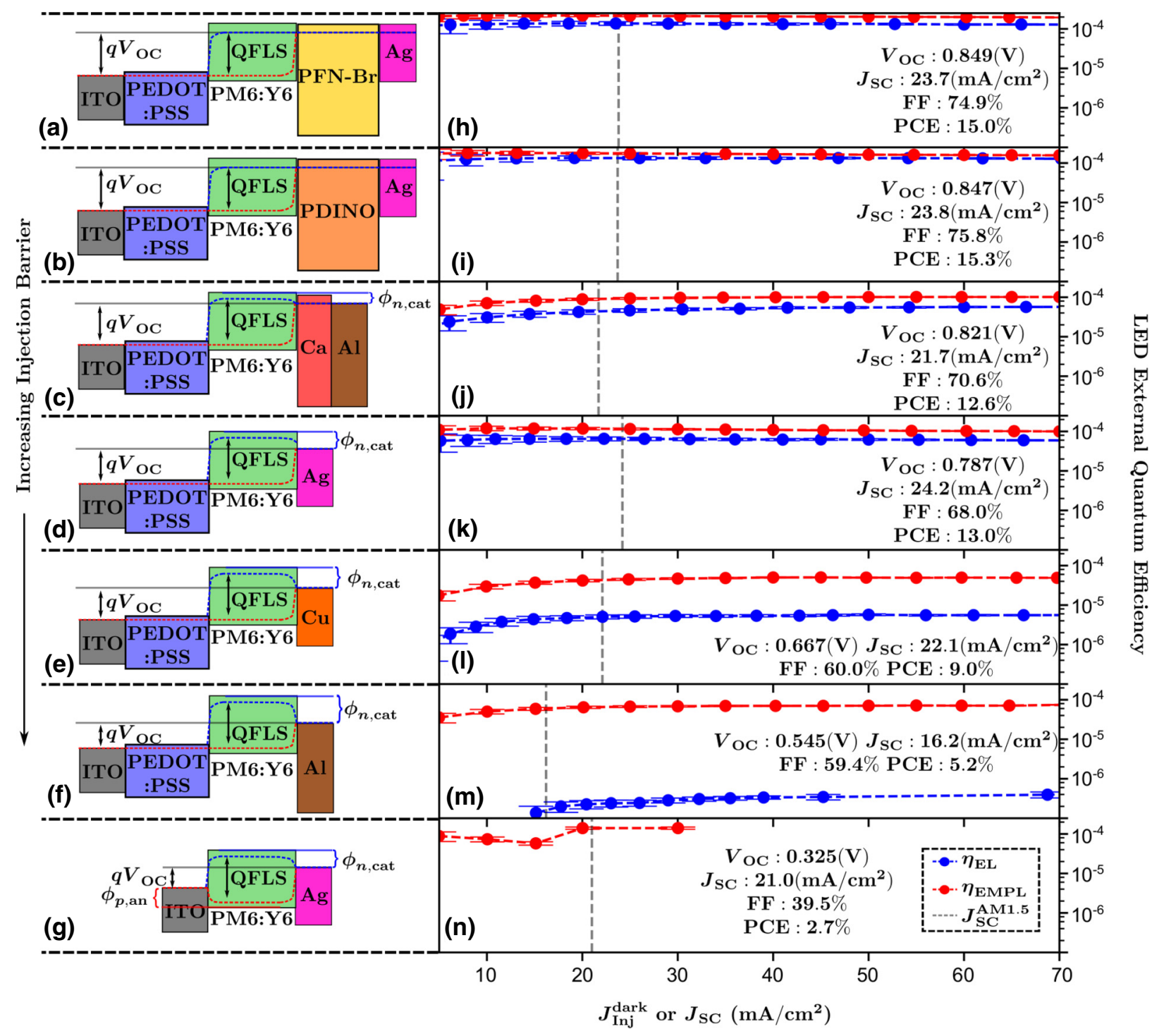

FIG. 4. (a)-(g) The device structures with an increasing injection barrier. (h)-(n) The LED external quantum efficiency as measured by electroluminescence $\left(\eta_{\mathrm{EL}}\right)$ as a function of the dark injected current (blue) and the electromodulated photoluminescence $\left(\eta_{\mathrm{EMPL}}\right)$ as a function of the short-circuit current (red) and $J_{\mathrm{SC}}^{\mathrm{AM} 1.5}$ (gray dashed line). PCE, power-conversion efficiency; FF, fill factor. The values of the device parameters and the measurements are taken from the top-performing pixel. The error bars are calculated from measurement errors in the oscilloscope and the lock-in amplifier (for details, see Sec. SIII of the Supplemental Material [47]).

electrode-induced losses are not obvious due to the experimental uncertainty. However, in the PFN-Br device $\left(V_{\mathrm{OC}}=0.849 \mathrm{~V}\right)$, the QFLS is $859 \pm 4 \mathrm{meV}$ while the electrode-induced losses account for a reduction in the $V_{\mathrm{OC}}$ of $10 \pm 4 \mathrm{meV}$. Improvements to the resolution will allow for discrepancies in the QFLS and the total nonradiative losses in an optimized cell to be distinguished, together quantifying the electrode-induced losses. This can be achieved by closely monitoring the temperature of the sample (accounting for about $3 \mathrm{meV}$ of error) and decreasing the measurement error in the lock-in amplifier or the oscilloscope (accounting for about $1 \mathrm{meV}$ of error each).

\section{CONCLUSIONS}

In conclusion, electromodulated-photoluminescence quantum yield is introduced as an approach to quantify the quasi-Fermi-level splitting in the bulk of organic photovoltaic devices under operational conditions. Simulated electromodulated-photoluminescence measurements are compared to traditional electroluminescence measurements, used to quantify the total nonradiative losses. It is shown that electroluminescence predicts the total nonradiative loss when the cathode is sufficiently Ohmic and varies substantially when it is not, while electromodulated photoluminescence accurately predicts the QFLS in the bulk of devices with both Ohmic and non-Ohmic cathodes. 


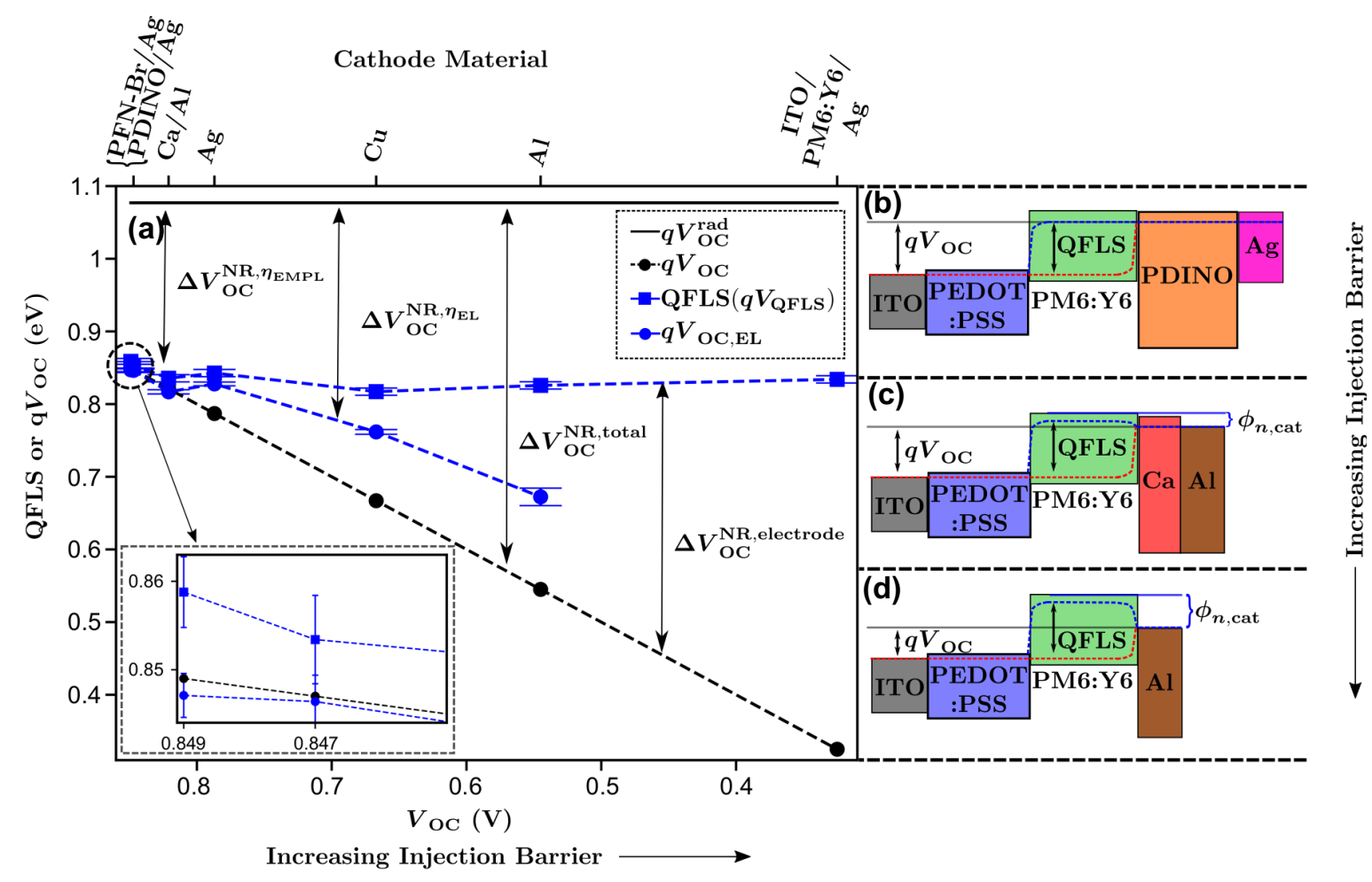

FIG. 5. (a) The QFLS measured by electromodulated photoluminescence (blue squares) and the expected $q V_{\mathrm{OC}}$ as measured by $\eta_{\mathrm{EL}}$ (blue circles) for PM6:Y6 systems with increasing electrode-induced nonradiative $V_{\mathrm{OC}}$ losses. The size of the various nonradiative losses to the $V_{\mathrm{OC}}$ is indicated on the plot. The upper axis lists cathode materials while the lower axis lists measured $V_{\mathrm{OC}}^{\mathrm{AM}} .5$. (b)- (d) The device structure designed to have (b) low, (c) medium, and (d) high nonradiative losses at the cathode.

PM6:Y6-based devices are fabricated with various cathodes and both electromodulated-photoluminescence and electroluminescence measurements are performed. The experiments show that with an increasingly non-Ohmic cathode, $\eta_{\mathrm{EL}}$ decreases dramatically while $\eta_{\mathrm{EMPL}}$ is unaffected, confirming the simulations. This shows that in the case of low-mobility systems, such as organic photovoltaic devices, $\eta_{\text {EMPL }}$ (along with the $V_{\mathrm{OC}}$ ) fully characterizes the different contributions of the nonradiative losses to the open-circuit voltage, providing a pathway to quantify the QFLS. In general, this method can be applied to devices based on any low-mobility semiconductor, as the simulations are agnostic to material type. It is found that in a high- $V_{\text {OC }}$ optimized solar cell, the QFLS is $859 \pm 4 \mathrm{meV}$, leading to the conclusion that the electrode-induced losses account for $10 \pm 4 \mathrm{mV}$ of the total photovoltage loss. This experimental technique provides a direct measurement of QFLS within organic solar cells and will contribute to the continual improvement of solar-cell and LED performance by allowing researchers to distinguish between intrinsic and electrode-induced losses to the open-circuit voltage.

\section{ACKNOWLEDGMENTS}

This work was supported by the Welsh Government's Sêr Cymru II Program through the European Regional
Development Fund, the Welsh European Funding Office, and the Swansea University strategic initiative in Sustainable Advanced Materials. A.A. is a Sêr Cymru II Rising Star Fellow and P.M. is a Sêr Cymru II National Research Chair. This work was also funded by U.K. Research and Innovation (UKRI) through the Engineering and Physical Sciences Research Council (EPSRC) Program Grant No. EP/T028511/1 Application Targeted Integrated Photovoltaics. D.B.R. acknowledges the support of the Natural Sciences and Engineering Research Council of Canada (NSERC) (Grant No. PGSD3-545694-2020). R.Ö. and N.M.W. would like to acknowledge support from Svenska tekniska vetenskapsakademien i Finland and The Society of Swedish Literature in Finland. R.Ö. acknowledges support from the Jane and Aatos Erkko foundation.

[1] Y. Lin, Y. Firdaus, F. H. Isikgor, M. I. Nugraha, E. Yengel, G. T. Harrison, R. Hallani, A. El-Labban, H. Faber, C. Ma, X. Zheng, A. Subbiah, C. T. Howells, O. M. Bakr, I. McCulloch, S. De Wolf, L. Tsetseris, and T. D. Anthopoulos, Self-assembled monolayer enables hole transport layer-free organic solar cells with $18 \%$ efficiency and improved operational stability, ACS Energy Lett. 5, 2935 (2020). 
[2] Q. Liu, Y. Jiang, K. Jin, J. Qin, J. Xu, W. Li, J. Xiong, J. Liu, Z. Xiao, K. Sun, S. Yang, X. Zhang, and L. Ding, $18 \%$ efficiency organic solar cells, Sci. Bull. 65, 272 (2020).

[3] Y. Cui, H. Yao, J. Zhang, T. Zhang, Y. Wang, L. Hong, K. Xian, B. Xu, S. Zhang, J. Peng, and Z. Wei, Over 16\% efficiency organic photovoltaic cells enabled by a chlorinated acceptor with increased open-circuit voltages, Nat. Commun. 10, 1 (2019).

[4] L. Perdigón-Toro, H. Zhang, A. Markina, J. Yuan, S. M. Hosseini, C. M. Wolff, G. Zuo, M. Stolterfoht, Y. Zou, F. Gao, D. Andrienko, S. Shoaee, and D. Neher, Barrierless free charge generation in the high-performance PM6:Y6 bulk heterojunction non-fullerene solar cell, Adv. Mater. 32, 1906763 (2020).

[5] A. Armin, W. Li, O. J. Sandberg, Z. Xiao, L. Ding, J. Nelson, D. Neher, K. Vandewal, S. Shoaee, T. Wang, H. Ade, T. Heumüller, C. Brabec, and P. Meredith, A history and perspective of non-fullerene electron acceptors for organic solar cells, Adv. Energy Mater. 11, 2003570 (2021).

[6] K. Vandewal, A. Gadisa, W. D. Oosterbaan, S. Bertho, F. Banishoeib, I. Van Severen, L. Lutsen, T. J. Cleij, D. Vanderzande, and J. V. Manca, The relation between opencircuit voltage and the onset of photocurrent generation by charge-transfer absorption in polymer: Fullerene bulk heterojunction solar cells, Adv. Funct. Mater. 18, 2064 (2008).

[7] K. Vandewal, K. Tvingstedt, A. Gadisa, O. Inganäs, and J. V. Manca, On the origin of the open-circuit voltage of polymer-fullerene solar cells, Nat. Mater. 8, 904 (2009).

[8] K. Vandewal, K. Tvingstedt, A. Gadisa, O. Inganäs, and J. V. Manca, Relating the open-circuit voltage to interface molecular properties of donor:acceptor bulk heterojunction solar cells, Phys. Rev. B 81, 125204 (2010).

[9] K. Vandewal, Z. Ma, J. Bergqvist, Z. Tang, E. Wang, P. Henriksson, K. Tvingstedt, Ma. R. Andersson, F. Zhang, and O. Inganäs, Quantification of quantum efficiency and energy losses in low bandgap polymer: Fullerene solar cells with high open-circuit voltage, Adv. Funct. Mater. 22, 3480 (2012).

[10] N. K. Elumalai and A. Uddin, Open circuit voltage of organic solar cells: An in-depth review, Energy Environ. Sci. 9, 391 (2016).

[11] D. Baran, T. Kirchartz, S. Wheeler, S. Dimitrov, M. Abdelsamie, J. Gorman, R. S. Ashraf, S. Holliday, A. Wadsworth, N. Gasparini, P. Kaienburg, H. Yan, A. Amassian, C. J. Brabec, J. R. Durrant, and I. McCulloch, Reduced voltage losses yield $10 \%$ efficient fullerene free organic solar cells with $>1$ V open circuit voltages, Energy Environ. Sci. 9, 3783 (2016).

[12] M. C. Scharber, D. Mühlbacher, M. Koppe, P. Denk, C. Waldauf, A. J. Heeger, and C. J. Brabec, Design rules for donors in bulk-heterojunction solar cells-Towards 10\% energy-conversion efficiency, Adv. Mater. 18, 789 (2006).

[13] V. D. Mihailetchi, P. W. M. Blom, J. C. Hummelen, and M. T. Rispens, Cathode dependence of the open-circuit voltage of polymer: Fullerene bulk heterojunction solar cells, J. Appl. Phys. 94, 6849 (2003).
[14] A. Wagenpfahl, C. Deibel, and V. Dyakonov, Organic solar cell efficiencies under the aspect of reduced surface recombination velocities, IEEE J. Sel. Top. Quantum Electron. 16, 1759 (2010).

[15] K. M. Knesting, H. Ju, C. W. Schlenker, A. J. Giordano, A. Garcia, O. L. Smith, D. C. Olson, S. R. Marder, and D. $\mathrm{S}$. Ginger, ITO interface modifiers can improve $V_{\text {oc }}$ in polymer solar cells and suppress surface recombination, J. Phys. Chem. Lett. 4, 4038 (2013).

[16] T. Kirchartz, F. Deledalle, P. S. Tuladhar, J. R. Durrant, and J. Nelson, On the differences between dark and light ideality factor in polymer: Fullerene solar cells, J. Phys. Chem. Lett. 4, 2371 (2013).

[17] J. Reinhardt, M. Grein, C. Bühler, M. Schubert, and U. Würfel, Identifying the impact of surface recombination at electrodes in organic solar cells by means of electroluminescence and modeling, Adv. Energy Mater. 4, 1400081 (2014).

[18] S. Boukli Hacène and T. Benouaz, Influence of charge carrier mobility and surface recombination velocity on the characteristics of P3HT:PCBM organic solar cells, Phys. Status Solidi (a) 211, 862 (2014).

[19] S. Wheeler, F. Deledalle, N. Tokmoldin, T. Kirchartz, J. Nelson, and J. R. Durrant, Influence of Surface Recombination on Charge-Carrier Kinetics in Organic Bulk Heterojunction Solar Cells with Nickel Oxide Interlayers, Phys. Rev. Appl. 4, 024020 (2015).

[20] I. Zonno, B. Krogmeier, V. Katte, D. Lübke, A. MartinezOtero, and T. Kirchartz, Discriminating between surface and bulk recombination in organic solar cells by studying the thickness dependence of the open-circuit voltage, Appl. Phys. Lett. 109, 183301 (2016).

[21] O. J. Sandberg and A. Armin, On the effect of surface recombination in thin film solar cells, light emitting diodes and photodetectors, Synth. Met. 254, 114 (2019).

[22] A. Karki, J. Vollbrecht, A. J. Gillett, S. Shuyong Xiao, Y. Yang, Z. Peng, N. Schopp, A. L. Dixon, S. Yoon, M. Schrock, H. Ade, G. N. Manjunatha Reddy, R. H. Friend, and T.-Q. Nguyen, The role of bulk and interfacial morphology in charge generation, recombination, and extraction in non-fullerene acceptor organic solar cells, Energy Environ. Sci. 13, 3679 (2020).

[23] W. Shockley, The theory of $p-n$ junctions in semiconductors and $p-n$ junction transistors, Bell Syst. Tech. J. 28, 435 (1949).

[24] P. Wurfel, The chemical potential of radiation, J. Phys. C: Solid State Phys. 15, 3967 (1982).

[25] I. L. Braly, D. W. deQuilettes, L. M. Pazos-Outón, S. Burke, M. E. Ziffer, D. S. Ginger, and H. W. Hillhouse, Hybrid perovskite films approaching the radiative limit with over $90 \%$ photoluminescence quantum efficiency, Nat. Photonics 12, 355 (2018).

[26] M. Stolterfoht, P. Caprioglio, C. M. Wolff, J. A. Márquez, J. Nordmann, S. Zhang, D. Rothhardt, U. Hörmann, Y. Amir, A. Redinger, L. Kegelmann, F. Zu, S. Albrecht, N. Koch, T. Kirchartz, M. Saliba, T. Unold, and D. Neher, The impact of energy alignment and interfacial recombination on the internal and external open-circuit voltage of perovskite solar cells, Energy Environ. Sci. 12, 2778 (2019). 
[27] Y. Cao, I. D. Parker, G. Yu C. Zhang, and A. J. Heeger, Improved quantum efficiency for electroluminescence in semiconducting polymers, Nature 397, 414 (1999).

[28] U. Mitschke and P. Bäuerle, The electroluminescence of organic materials, J. Mater. Chem. 10, 1471 (2000).

[29] A. C. Grimsdale, K. Leok Chan, R. E. Martin, P. G. Jokisz, and A. B. Holmes, Synthesis of light-emitting conjugated polymers for applications in electroluminescent devices, Chem. Rev. 109, 897 (2009).

[30] A. A. Bakulin, A. Rao, V. G. Pavelyev, P. H. M. van Loosdrecht, M. S. Pshenichnikov, D. Niedzialek, J. Cornil, D. Beljonne, and R. H. Friend, The role of driving energy and delocalized states for charge separation in organic semiconductors, Science 335, 1340 (2012).

[31] A. Classen, C. L. Chochos, L. Lüer, V. G. Gregoriou, J. Wortmann, A. Osvet, K. Forberich, I. McCulloch, T. Heumüller, and C. J. Brabec, The role of exciton lifetime for charge generation in organic solar cells at negligible energy-level offsets, Nat. Energy 5, 711 (2020).

[32] U. Rau, Reciprocity relation between photovoltaic quantum efficiency and electroluminescent emission of solar cells, Phys. Rev. B 76, 085303 (2007).

[33] M. A. Green, J. Zhao, A. Wang, P. J. Reece, and M. Gal, Efficient silicon light-emitting diodes, Nature 412, 805 (2001).

[34] T. Kirchartz and U. Rau, Electroluminescence analysis of high efficiency $\mathrm{Cu}(\mathrm{In}, \mathrm{Ga}) \mathrm{Se}_{2}$ solar cells, J. Appl. Phys. 102, 104510 (2007).

[35] T. Kirchartz, U. Rau, M. Hermle, A. W. Bett, A. Helbig, and J. H. Werner, Internal voltages in $\mathrm{GaInP} / \mathrm{GaInAs} / \mathrm{Ge}$ multijunction solar cells determined by electroluminescence measurements, Appl. Phys. Lett. 92, 123502 (2008).

[36] T. Kirchartz, A. Helbig, W. Reetz, M. Reuter, J. H. Werner, and U. Rau, Reciprocity between electroluminescence and quantum efficiency used for the characterization of silicon solar cells, Prog. Photovoltaics: Res. Appl. 17, 394 (2009).

[37] O. J. Sandberg, A. Sundqvist, M. Nyman, and R. Österbacka, Relating Charge Transport, Contact Properties, and Recombination to Open-Circuit Voltage in Sandwich-Type Thin-Film Solar Cells, Phys. Rev. Appl. 5, 044005 (2016).

[38] A. Spies, M. List, T. Sarkar, and U. Würfel, On the impact of contact selectivity and charge transport on the open-circuit voltage of organic solar cells, Adv. Energy Mater. 7, 1601750 (2017).

[39] L. Q. Phuong, S. M. Hosseini, O. J. Sandberg, Y. Zou, H. Y. Woo, D. Neher, and S. Shoaee, Quantifying quasi-Fermi level splitting and open-circuit voltage losses in highly efficient nonfullerene organic solar cells, Solar RRL, 5, 2000649 (2020).

[40] D. L. Scharfetter and H. K. Gummel, Large-signal analysis of a silicon read diode oscillator, IEEE Trans. Electron Devices 16, 64 (1969).

[41] S. Selberherr, Analysis and Simulation of Semiconductor Devices (Springer Science \& Business Media, Berlin/Heidelberg, Germany, 2012).

[42] L. J. A. Koster, E. C. P. Smits, V. D. Mihailetchi, and P. W. M. Blom, Device model for the operation of polymer/fullerene bulk heterojunction solar cells, Phys. Rev. B 72, 085205 (2005).

[43] P. Würfel and U. Würfel, Physics of Solar Cells: From Basic Principles to Advanced Concepts (John Wiley \& Sons, Hoboken, NJ, 2016).

[44] M. Azzouzi, J. Yan, T. Kirchartz, K. Liu, J. Wang, H. $\mathrm{Wu}$, and J. Nelson, Nonradiative Energy Losses in BulkHeterojunction Organic Photovoltaics, Phys. Rev. X 8, 031055 (2018).

[45] E. H. Rhoderick, Metal-semiconductor contacts, IEE Proc. I-Solid-State Electron Devices, 129, 1 (1982).

[46] A. Wagenpfahl, D. Rauh, M. Binder, C. Deibel, and V. Dyakonov, S-shaped current-voltage characteristics of organic solar devices, Phys. Rev. B 82, 115306 (2010).

[47] See the Supplemental Material at http://link.aps.org/supple mental/10.1103/PhysRevApplied.15.064035 for further information on drift-diffusion simualtions, device fabrication, data analysis, error calculation, and list of the chemical acronyms.

[48] N. Zarrabi, O. J. Sandberg, S. Zeiske, W. Li, D. B. Riley, P. Meredith, and A. Armin, Charge-generating mid-gap trap states define the thermodynamic limit of organic photovoltaic devices, Nat. Commun. 11, 5567 (2020).

[49] O. J. Sandberg, M. Nyman, and R. Österbacka, Effect of Contacts in Organic Bulk Heterojunction Solar Cells, Phys. Rev. Appl. 1, 024003 (2014). 